IP Selected for a Viewpoint in Physics

PHYSICAL REVIEW A 82, 011605(R) (2010)

\title{
Phases of a bilayer Fermi gas
}

\author{
Yusuke Nishida \\ Center for Theoretical Physics, Massachusetts Institute of Technology, Cambridge, Massachusetts 02139, USA
}

(Received 6 July 2009; published 12 July 2010)

\begin{abstract}
We investigate a two-species Fermi gas in which one species is confined in two parallel layers and interacts with the other species in the three-dimensional space by a tunable short-range interaction. Based on the controlled weak coupling analysis and the exact three-body calculation, we show that the system has a rich phase diagram in the plane of the effective scattering length and the layer separation. Resulting phases include an interlayer $s$-wave pairing, an intralayer $p$-wave pairing, a dimer Bose-Einstein condensation, and a Fermi gas of stable Efimov-like trimers. Our system provides a widely applicable scheme to induce long-range interlayer correlations in ultracold atoms.
\end{abstract}

DOI: 10.1103/PhysRevA.82.011605

PACS number(s): 03.75.Ss, 05.30.Fk, 74.20.Rp, 74.78.-w

\section{INTRODUCTION}

One of the central themes in ultracold atoms is to provide highly tunable model systems for other subfields in physics. A prominent example is the realization of ultracold twodimensional atomic gases which have enabled detailed microscopic studies of Berezinskii-Kosterlitz-Thouless physics relevant to a wide variety of two-dimensional phenomena [1-3]. In addition to simple two-dimensional systems with single layers, what have attracted considerable attention in condensed matter physics are bilayer or multilayer systems. Here, extra degrees of freedom generated by layers and the long-range Coulomb interaction between them are expected to lead to intriguing physics such as an interlayer exciton condensation in bilayer semiconductors [4,5], quantum Hall bilayers [6], and bilayer graphenes [7].

In ultracold atoms, the analogous multilayer geometry can be created by confining atoms by a strong optical lattice in one direction. However, since the long-range Coulomb interaction is absent in neutral atoms, separated layers are simply decoupled without interlayer tunneling. A scheme that we propose in this article to realize the long-range interlayer correlation in ultracold atoms is to use a mixture of two atomic species $A$ and $B$ and confine only $A$ atoms in the multilayer geometry with keeping $B$ atoms in the three-dimensional space. Here the correlation between $A$ atoms confined in different layers can be induced through the interaction with the background $B$ atoms that are free to propagate from layer to layer. The advantage of this scheme is that one can tune the $A-B$ interaction by interspecies Feshbach resonances and thus rich phenomena are expected to occur as a function of the interaction strength. Furthermore, such a system may be thought of as a tunable model system for a slab phase of nuclear matter by identifying $A(B)$ atoms with protons (neutrons) [8,9].

In this article, we give detailed analyses in the case of a bilayer Fermi gas that can be realized by using a Fermi-Fermi mixture of, for example, ${ }^{6} \mathrm{Li}$ and ${ }^{40} \mathrm{~K}$ [10-12] and confining only one species in two parallel layers. Based on the controlled weak coupling analysis and the exact three-body calculation, we show that the system exhibits at least four distinct quantum phases as summarized in Fig. 1. In the weak coupling region, an effective attraction between two $A$ atoms mediated by $B$ atoms leads to superfluidity due to an interlayer $s$-wave pairing (lower left phase) or an intralayer $p$-wave pairing (upper left phase) depending on the layer separation. On the other hand, in the strong coupling region, an $A$ atom captures a $B$ atom to form a tightly bound molecule and the ground state becomes a dimer Bose-Einstein condensation (right phase). Finally, when the $A-B$ interaction is close to the resonance, we find that two $A$ atoms confined in different layers with one $B$ atom form a three-body bound state leading to a Fermi gas of trimers in a dilute system (lower middle phase). Remarkably, there is an infinite number of such three-body bound levels exactly at the resonance resembling the Efimov effect in three dimensions [13].

The system under consideration is described by the action (here and below $\hbar=1$ and $k_{\mathrm{B}}=1$ ):

$$
\begin{aligned}
S= & \sum_{i=1,2} \int d t d \boldsymbol{x} \psi_{A i}^{\dagger}(t, \boldsymbol{x})\left(i \partial_{t}+\frac{\nabla_{\boldsymbol{x}}^{2}}{2 m_{A}}+\mu_{A}\right) \psi_{A i}(t, \boldsymbol{x}) \\
& +\int d t d \boldsymbol{x} d z \psi_{B}^{\dagger}(t, \boldsymbol{x}, z)\left(i \partial_{t}+\frac{\nabla_{\boldsymbol{x}}^{2}+\nabla_{z}^{2}}{2 m_{B}}+\mu_{B}\right)
\end{aligned}
$$

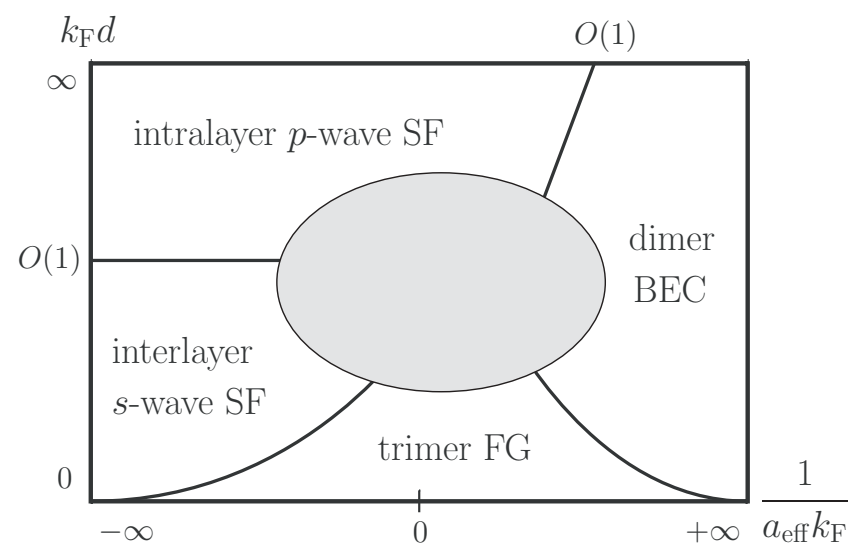

FIG. 1. Conjectured phase diagram at zero temperature in the plane of the effective scattering length $\left(a_{\mathrm{eff}} k_{\mathrm{F}}\right)^{-1}$ and the layer separation $k_{\mathrm{F}} d$. Here, $k_{\mathrm{F}} \sim k_{\mathrm{FA}} \sim k_{\mathrm{FB}}$ is assumed. 


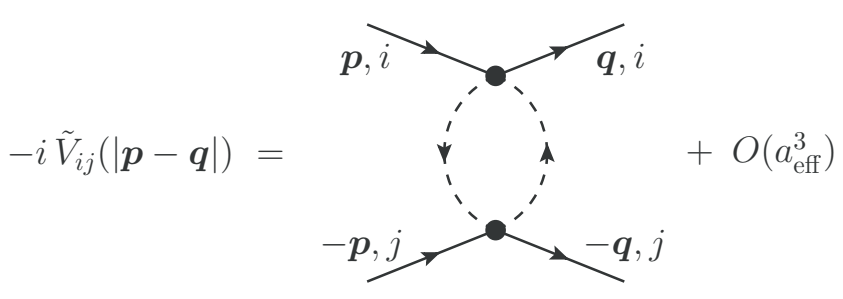

FIG. 2. Effective interaction between two $A$ atoms (solid line) induced by the interaction with $B$ atoms (dotted line) in the weak coupling limit. Here, $i, j=1,2$ are layer indices.

$$
\begin{aligned}
& \times \psi_{B}(t, \boldsymbol{x}, z)+g_{0} \sum_{i=1,2} \int d t d \boldsymbol{x} \psi_{A i}^{\dagger}(t, \boldsymbol{x}) \psi_{B}^{\dagger}\left(t, \boldsymbol{x}, z_{i}\right) \\
& \times \psi_{B}\left(t, \boldsymbol{x}, z_{i}\right) \psi_{A i}(t, \boldsymbol{x}) .
\end{aligned}
$$

Here, $\psi_{A i}(t, \boldsymbol{x})$ with $\boldsymbol{x}=(x, y)$ is a spinless fermionic field for $A$ atoms confined in two parallel layers that are labeled by the index $i=1,2$ and located at $z=z_{i} . \psi_{B}(t, \boldsymbol{x}, z)$ is another spinless fermionic field for $B$ atoms in the three-dimensional space. The last term in the action describes the short-range $A-B$ interaction. $g_{0}$ is a cutoff $(\Lambda)$ dependent bare coupling, which can be eliminated by introducing the physical parameter, the effective scattering length $a_{\text {eff }}$, through $\frac{1}{g_{0}}-\frac{\sqrt{m_{B} m_{A B}}}{2 \pi} \Lambda=$ $-\frac{\sqrt{m_{B} m_{A B}}}{2 \pi a_{\text {eff }}}$ with $m_{A B} \equiv m_{A} m_{B} /\left(m_{A}+m_{B}\right)$ being the reduced mass [14,15]. $a_{\text {eff }}$ is arbitrarily tunable by means of the interspecies Feshbach resonance and the limit $a_{\text {eff }} \rightarrow-(+) 0$ corresponds to the weak (strong) attraction between $A$ and $B$ atoms. Throughout this article, the interlayer tunneling is assumed to be negligible and Fermi momenta of $A$ and $B$ atoms are defined through their densities; $k_{\mathrm{FA}} \equiv\left(4 \pi n_{A}\right)^{1 / 2}$ and $k_{\mathrm{FB}} \equiv\left(6 \pi^{2} n_{B}\right)^{1 / 3}$.

\section{WEAK COUPLING LIMIT}

In order to elucidate phases appearing in our system, we start with the weak coupling limit $a_{\text {eff }} \rightarrow-0$ in which a controlled perturbative analysis is possible. Unlike the ordinary BCS-BEC crossover purely in two or three dimensions, the $A-B$ pairing at weak coupling does not take place in our mixed dimensional system because of the absence of the Cooper instability [15]. What can happen instead are pairings between $A$ atoms using the effective attraction induced by the interaction with the Fermi sea of $B$ atoms. To the leading order in $a_{\text {eff }}$, the back-to-back scattering of $A$ atoms in layers $i$ and $j$ is described by the Feynman diagram in Fig. 2. The resulting induced interaction $\tilde{V}_{i j}(|\boldsymbol{p}-\boldsymbol{q}|)$ has rather a simple form in the real space:

$$
V_{i j}(|\boldsymbol{x}|)=\frac{a_{\mathrm{eff}}^{2}}{m_{A B}} \frac{2 k_{\mathrm{FB}} r_{i j} \cos \left(2 k_{\mathrm{FB}} r_{i j}\right)-\sin \left(2 k_{\mathrm{FB}} r_{i j}\right)}{4 \pi r_{i j}^{4}},
$$

where $r_{i j}=\sqrt{\boldsymbol{x}^{2}+\left(z_{i}-z_{j}\right)^{2}}$ is the distance between the two $A$ atoms. The oscillatory decaying factor is well known in the Ruderman-Kittel-Kasuya-Yosida interaction [16-18] and leads to the following physical interpretation of $V_{i j}(|\boldsymbol{x}|)$ : The density modulation of background $B$ atoms produced by one $A$ atom in the layer $i$ mediates the long-range interaction with the other $A$ atom in the layer $j$.

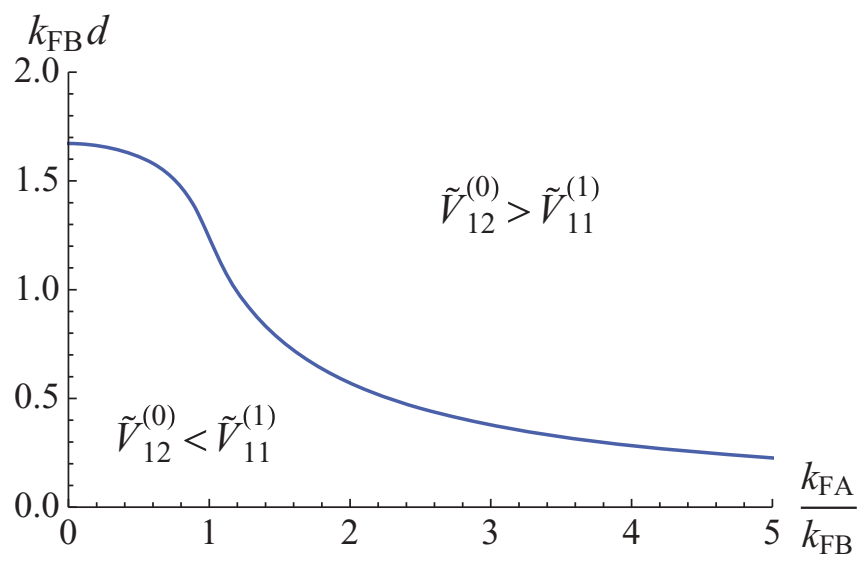

FIG. 3. (Color online) Critical layer separation $k_{\mathrm{FB}} d$ dividing the interlayer $s$-wave pairing $\left(\tilde{V}_{12}^{(0)}<\tilde{V}_{11}^{(1)}\right)$ from the intralayer $p$-wave pairing $\left(\tilde{V}_{12}^{(0)}>\tilde{V}_{11}^{(1)}\right)$ as a function of $k_{\mathrm{FA}} / k_{\mathrm{FB}}$.

Now the pairings of $A$ atoms are described by the BCS-type Hamiltonian:

$$
\begin{aligned}
H_{\text {ind }}= & \sum_{i} \sum_{\boldsymbol{p}} \tilde{\psi}_{A i}^{\dagger}(\boldsymbol{p})\left(\frac{\boldsymbol{p}^{2}}{2 m_{A}}-\mu_{A}\right) \tilde{\psi}_{A i}(\boldsymbol{p}) \\
& +\frac{1}{2 \Omega} \sum_{i, j} \sum_{\boldsymbol{p}, \boldsymbol{q}} \tilde{\psi}_{A i}^{\dagger}(\boldsymbol{p}) \tilde{\psi}_{A j}^{\dagger}(-\boldsymbol{p}) \\
& \times \tilde{V}_{i j}(|\boldsymbol{p}-\boldsymbol{q}|) \tilde{\psi}_{A j}(-\boldsymbol{q}) \tilde{\psi}_{A i}(\boldsymbol{q}) .
\end{aligned}
$$

In the mean-field approximation, the gap function is given by $\Delta_{i j}(\boldsymbol{p})=\frac{1}{\Omega} \sum_{\boldsymbol{q}} \tilde{V}_{i j}(|\boldsymbol{p}-\boldsymbol{q}|)\left\langle\tilde{\psi}_{A j}(-\boldsymbol{q}) \tilde{\psi}_{A i}(\boldsymbol{q})\right\rangle$ and the Fermi statistics of $A$ atoms implies $\Delta_{j i}(-\boldsymbol{p})=-\Delta_{i j}(\boldsymbol{p})$. Although $A$ atoms are spinless fermions, the layer indices $i, j=1,2$ make more than one pairing pattern possible. When the layer separation is large $d \equiv\left|z_{1}-z_{2}\right| \rightarrow \infty$, the interlayer interaction $V_{i \neq j}$ is suppressed while the intralayer interaction $V_{i=j}$ is unaffected by $d$. Therefore, in this limit, intralayer pairings with $\Delta_{11}, \Delta_{22} \neq 0$ are favored while their pairing symmetry has to be $p$ wave [15]. On the other hand, when the layer separation becomes small $d \rightarrow 0, V_{i \neq j}$ is no longer suppressed and thus an interlayer pairing with $\Delta_{12}=-\Delta_{21} \neq$ 0 is favored because the pairing symmetry can be $s$ wave only in this singlet channel. Therefore, there has to be a quantum phase transition as a function of the layer separation.

The quantum phase transition can be located by comparing the energy densities for the interlayer $s$-wave and intralayer $p$-wave pairings. In the weak coupling limit, their energy densities are, respectively, given by $\left\langle\mathcal{H}_{\text {ind }}\right\rangle=-\frac{m_{A} \mu_{A}^{2}}{2 \pi}-$ $\frac{m_{A}\left|\Delta_{12}\right|^{2}}{4 \pi}$ and $\left\langle\mathcal{H}_{\text {ind }}\right\rangle=-\frac{m_{A} \mu_{A}^{2}}{2 \pi}-\frac{m_{A}\left|\Delta_{11}\right|^{2}}{8 \pi}-\frac{m_{A}\left|\Delta_{22}\right|^{2}}{8 \pi}$, where the gap functions at $|\boldsymbol{p}|=k_{\mathrm{FA}}$ are given by $\Delta_{12} / \varepsilon_{\mathrm{FA}} \propto e^{2 \pi /\left[m_{A} \tilde{V}_{12}^{(0)}\right]}$ and $\Delta_{11} / \varepsilon_{\mathrm{FA}}=\Delta_{22} / \varepsilon_{\mathrm{FA}} \propto\left(\hat{p}_{x} \pm i \hat{p}_{y}\right) e^{2 \pi /\left[m_{A} \tilde{V}_{11}^{(1)}\right]}$ for negative $\tilde{V}_{i j}^{(l)}$. Here, $\varepsilon_{\mathrm{FA}} \equiv k_{\mathrm{FA}}^{2} /\left(2 m_{A}\right)$ is the Fermi energy and $\tilde{V}_{i j}^{(l)} \equiv$ $\int_{0}^{\pi} \frac{d \varphi}{\pi} \cos (l \varphi) \tilde{V}_{i j}(|\boldsymbol{p}-\boldsymbol{q}|)$ with $\cos \varphi \equiv \hat{\boldsymbol{p}} \cdot \hat{\boldsymbol{q}}$ is the partial wave projection of the induced interaction in which both incoming and outgoing momenta are restricted on the Fermi surface of $A$ atoms; $|\boldsymbol{p}|=|\boldsymbol{q}|=k_{\mathrm{F} A}$. It is the phase with the smaller energy density and hence the larger pairing gap that is realized in our system.

The critical layer separation dividing the above two phases is plotted in Fig. 3 as a function of $k_{\mathrm{FA}} / k_{\mathrm{FB}}$, which is 

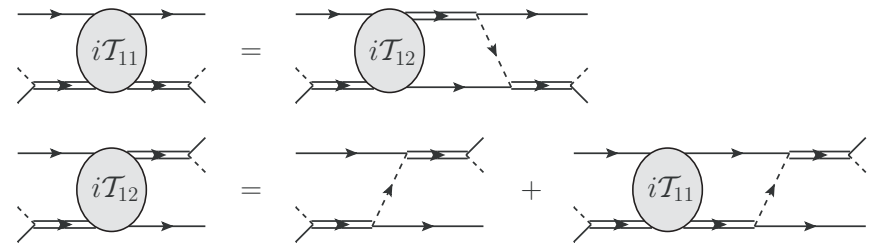

FIG. 4. Three-body scattering of two $A$ atoms in different layers and one $B$ atom. The double line represents the $A-B$ scattering amplitude. $\mathcal{T}_{22}$ and $\mathcal{T}_{21}$ satisfy the same integral equations as $\mathcal{T}_{11}$ and $\mathcal{T}_{12}$.

independent of $a_{\text {eff }}$ and $m_{A} / m_{B}$ to the leading order in $a_{\text {eff }}$. Below the critical separation where $\tilde{V}_{12}^{(0)}<\tilde{V}_{11}^{(1)}$, the interlayer $s$-wave pairing appears, while the intralayer $p$-wave pairing appears above the critical separation where $\tilde{V}_{12}^{(0)}>\tilde{V}_{11}^{(1)}$. The two layers exhibit superfluidity in both phases and its critical temperature at weak coupling is the same order as the pairing gap at zero temperature; $T_{\mathrm{c}} \sim\left|\Delta_{i j}\right|$. We note that our interlayer $s$-wave pairing bears an analogy to the interlayer exciton condensation in condensed matter systems [4-7]. Also it is worthwhile to point out that the intralayer $p_{x}+i p_{y}$-wave pairing has a potential application to topological quantum computation using vortices with non-Abelian statistics $[19,20]$.

\section{STRONG COUPLING LIMIT}

We now turn to the strong coupling limit $a_{\text {eff }} \rightarrow+0$, in which $A$ atoms confined in layers capture $B$ atoms from the bulk space to form two-body bound states (dimers) whose binding energy is given by $E_{2}=-\frac{1}{2 m_{A B} a_{\mathrm{eff}}^{2}}$. The resulting system consists of the dimers localized around layers which interact weakly with each other and excess $B$ atoms. As long as the dimer size $\sim a_{\text {eff }}$ is smaller than the layer separation $d$ and the mean interparticle distance $\sim k_{\mathrm{FA}}^{-1}$, the dimers behave as two-dimensional bosons and therefore the ground state becomes a dimer Bose-Einstein condensation in each layer. Accordingly, the two layers exhibit superfluidity up to the Berezinskii-Kosterlitz-Thouless temperature given by $T_{\mathrm{BKT}} \rightarrow \frac{2 \pi n_{d}}{M} \ln ^{-1}\left(-\frac{380}{4 \pi} \ln n_{d} a_{\mathrm{eff}}^{2}\right)$ in the limit $a_{\mathrm{eff}} \rightarrow+0$ [21], where $M \equiv m_{A}+m_{B}$ and $n_{d}=n_{A}$ are the dimer's mass and density per layer. We note that the dimer Bose-Einstein condensates in different layers can interact with each other through the excess $B$ atoms just as in Fig. 2 and Eq. (2).

Although our system is found to exhibit superfluidity in both weak and strong coupling limits $a_{\text {eff }} \rightarrow \mp 0$, their symmetry breaking patterns are different. The action in Eq. (1) has continuous symmetries of $\mathrm{U}(1)_{A 1} \times \mathrm{U}(1)_{A 2} \times \mathrm{U}(1)_{B} \times \mathrm{U}(1)_{z}$ corresponding to particle number conservations of $A$ atoms in each layer, that of $B$ atoms, and a rotation about $z$ axis. An order parameter of the interlayer $s$-wave pairing $\left\langle\epsilon_{i j} \psi_{A i} \psi_{A j}\right\rangle \neq 0$ breaks the full symmetries down to $\mathrm{U}(1)_{A 1-A 2} \times \mathrm{U}(1)_{B} \times$ $\mathrm{U}(1)_{z}$, while order parameters of the intralayer $p_{x}+i p_{y^{-}}$ wave pairing $\left\langle\psi_{A i}\left(\stackrel{\leftrightarrow}{\partial}_{x}+i \stackrel{\leftrightarrow}{\partial}_{y}\right) \psi_{A i}\right\rangle \neq 0$ break them down to $\mathrm{U}(1)_{A 1+A 2-z} \times \mathrm{U}(1)_{B}$. Order parameters of the dimer BoseEinstein condensation are $\left\langle\psi_{A 1} \psi_{B}\right\rangle,\left\langle\psi_{A 2} \psi_{B}\right\rangle \neq 0$, which break the full symmetries down to $\mathrm{U}(1)_{A 1+A 2-B} \times \mathrm{U}(1)_{z}$. Therefore, the phases in the weak and strong coupling limits have to be divided by at least one quantum phase transition as

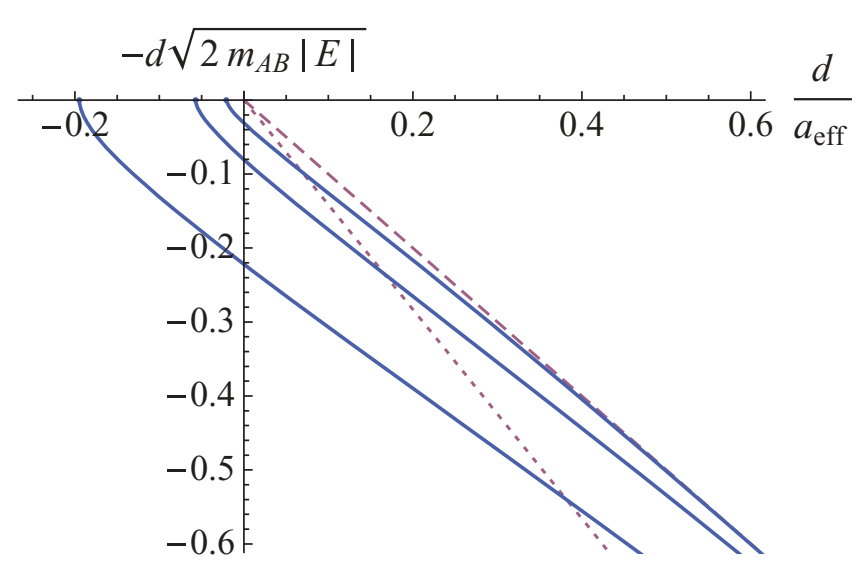

FIG. 5. (Color online) Lowest binding energies of trimers for mass ratios $m_{A} / m_{B}=6.67$ (bottom), 1 (middle), and 0.15 (top) as a function of $d / a_{\text {eff }}$. The dashed and dotted lines are atom-dimer and dimer-dimer thresholds; $E=E_{2}$ and $2 E_{2}$.

a function of the interaction strength. Below we show that a novel phase can appear in between when the layer separation is small.

\section{UNITARITY LIMIT}

The fourth phase realized in our system can be elucidated by studying a three-body problem of two $A$ atoms confined in different layers interacting with one $B$ atom. Their scattering process is depicted in Fig. 4 and all the relevant diagrams can be summed by solving the integral equations for $\mathcal{T}_{i j}(E ; \boldsymbol{p}, \boldsymbol{q})$. Here $E$ is the total energy in the center-of-mass frame and $\boldsymbol{p}(\boldsymbol{q})$ is the relative momentum of the incoming (outgoing) $A$ atom in the layer $i(j)$ that does not scatter with the $B$ atom first (last). The $\mathcal{T}$-matrix elements have properties $\mathcal{T}_{11}=$ $\mathcal{T}_{22}$ and $\mathcal{T}_{12}=\mathcal{T}_{21}$ and can be decomposed into even- and odd-parity parts under the exchange of one layer index; $\mathcal{T}_{ \pm} \equiv$ $\mathcal{T}_{11} \pm \mathcal{T}_{12}$. After the partial wave projection $\mathcal{T}_{ \pm}^{(l)}(E ; p, q) \equiv$ $\int_{0}^{\pi} \frac{d \varphi}{\pi} \cos (l \varphi) \mathcal{T}_{ \pm}(E ; \boldsymbol{p}, \boldsymbol{q})$ with $\cos \varphi \equiv \hat{\boldsymbol{p}} \cdot \hat{\boldsymbol{q}}$, we find $\mathcal{T}_{ \pm}^{(l)}$ to satisfy

$$
\begin{aligned}
\mathcal{T}_{ \pm}^{(l)}(E ; p, q) & \\
= & \mp \sqrt{m_{B} m_{A B}} K^{(l)}\left(E+i 0^{+} ; p, q\right) \\
& \mp \int_{0}^{\infty} d k k \frac{\mathcal{T}_{ \pm}^{(l)}(E ; p, k) K^{(l)}\left(E+i 0^{+} ; k, q\right)}{\sqrt{\frac{m_{B}+m_{A B}}{M} k^{2}-2 m_{A B} E-i 0^{+}}-\frac{1}{a_{\mathrm{eff}}}}
\end{aligned}
$$

where $K^{(l)}(E ; p, q)$ is given by

$$
\int_{0}^{\pi} \frac{d \varphi}{\pi} \cos (l \varphi) \frac{e^{-d \sqrt{\frac{M}{m_{A}}} \sqrt{p^{2}+q^{2}+\frac{2 m_{A}}{M} p q \cos \varphi-2 m_{A B} E}}}{\sqrt{p^{2}+q^{2}+\frac{2 m_{A}}{M} p q \cos \varphi-2 m_{A B} E}} .
$$

The spectrum of three-body bound states (trimers) is obtained by poles of $\mathcal{T}_{ \pm}^{(l)}$ as a function of $E$. When $E$ approaches one of the binding energies $E_{3}<-\frac{\theta\left(a_{\mathrm{eff}}\right)}{2 m_{A B} a_{\mathrm{eff}}^{2}}$, we can write $\mathcal{T}_{ \pm}^{(l)}$ as $\mathcal{T}_{ \pm}^{(l)}(E ; p, q) \rightarrow \mathcal{Z}_{ \pm}^{(l)}(p, q) /\left(E-E_{3}\right)$. By solving the homogeneous integral equation from Eq. (4) satisfied by the residue $\mathcal{Z}_{ \pm}^{(l)}$, we find that the trimers exist only in an odd-parity $(-)$ and $s$-wave $(l=0)$ channel. The lowest binding energy $E_{3}^{(0)}$ as a function of $d / a_{\text {eff }}$ is plotted in Fig. 5 for 
three different values of the mass ratio $m_{A} / m_{B}=0.15,1$, and 6.67, corresponding to a mixture of $A={ }^{6} \mathrm{Li}$ and $B={ }^{40} \mathrm{~K}$, two different internal states of the same atomic species, and $A={ }^{40} \mathrm{~K}$ and $B={ }^{6} \mathrm{Li}$. The trimer appears on the negative side of the effective scattering length $\left|a_{\text {eff }}\right| \sim d$ and its binding energy decreases as $d / a_{\text {eff }}$ is increased. When $d / a_{\text {eff }} \rightarrow+\infty$, the trimer binding energy asymptotically approaches the atom-dimer threshold $E=E_{2}$ from below as $E_{3} / E_{2}-1 \propto$ $e^{-\frac{m_{B}+m_{A B}}{M} \exp \left(\sqrt{\frac{M}{m_{A}}} \frac{d}{a_{\text {eff }}}\right)} \rightarrow+0$.

Interestingly, when the $A-B$ interaction is exactly at the twobody resonance $\left|a_{\text {eff }}\right| \rightarrow \infty$, there exists an infinite number of such trimer states whose spectrum is expressed by the form $E_{3}^{(n)}=-e^{-2 \pi n / s_{0}} \frac{\kappa^{2}}{2 m_{A B} d^{2}}$ for $n \rightarrow \infty$ (not shown in Fig. 5). Such a geometric spectrum at the resonance is well known as the Efimov effect in three dimensions [13]. Here the scaling exponent $s_{0}$ and the so-called Efimov parameter $\kappa$ can be determined as $\left(s_{0}, \kappa\right)=(0.741,0.0311),(0.828,0.0807)$, $(1.30,0.231)$ for $m_{A} / m_{B}=0.15,1,6.67$, respectively. An important characteristic of our trimer state in contrast to Efimov trimers in a free space is its stability against the threebody recombination because the two $A$ atoms are spatially separated.

The existence of stable $A A B$ trimers, which has the same quantum number as $\epsilon_{i j} \psi_{A i} \psi_{A j} \psi_{B}$, can lead to a Fermi gas of trimers in a dilute system. As long as the trimer size $\left(2 m_{A B}\left|E_{3}\right|\right)^{-1 / 2} \sim d$ is smaller than the mean interparticle distance $\sim k_{\mathrm{FA}}^{-1}$, the trimers behave as two-dimensional fermions localized around layers and form a Fermi gas with its density equal to $n_{A}$. Although the trimer state exists even in the strong coupling limit $a_{\text {eff }} \rightarrow+0$, the trimer gas phase cannot persist there because an addition of another $B$ atom breaks up the trimer into two dimers when $E_{3}>2 E_{2}$ is reached (see the dimer-dimer threshold in Fig. 5). Therefore, the trimer Fermi gas is realized only when the $A-B$ interaction is close to the resonance $\left|a_{\text {eff }}\right| \gtrsim d$. We note that $A A B$ trimers with $A$ atoms in the same layer and $A B B$ trimers are absent for $0.0351<m_{A} / m_{B}<6.35$ [14]. Once they are formed, the whole phase diagram would be dominated by such deeply bound trimers whose size is set by the thickness of layers.

\section{CONCLUSIONS}

We found that the dimer Bose-Einstein condensation appears in the strong coupling region where $a_{\text {eff }} \lesssim d$ and $a_{\mathrm{eff}} k_{\mathrm{FA}} \lesssim O(1)$, while the trimer Fermi gas appears in the unitarity region where $\left|a_{\mathrm{eff}}\right| \gtrsim d$ and $k_{\mathrm{FA}} d \lesssim O(1)$. Assuming that the rest of the phase diagram is occupied by the interlayer $s$-wave or intralayer $p$-wave superfluidity found in the weak coupling region, we conclude that the system has the rich phase diagram shown in Fig. 1. Our results can be tested by using a ultracold Fermi-Fermi mixture of ${ }^{6} \mathrm{Li}$ and ${ }^{40} \mathrm{~K}$ with a species-selective optical lattice. How the above four phases meet at the center of the phase diagram (shaded region in Fig. 1) and whether more quantum phases appear in our system are interesting open questions and can be addressed, in principle, by future experiments. In particular, further study on how the dilute gas of trimers evolves into a gas of their constituents as $k_{\mathrm{FA}} d$ is increased may shed light on a similar problem of the transition from nuclear matter to quark matter existing in the core of neutron stars. Finally, we emphasize that the scheme to induce long-range interlayer correlations in ultracold atoms that is presented in this article by taking the bilayer Fermi gas as an example can be widely extended to multilayer geometries, multiwire geometries, Bose-Bose mixtures, and Bose-Fermi mixtures.

\section{ACKNOWLEDGMENTS}

Y. N. is supported by MIT Pappalardo Fellowship in Physics and the US Department of Energy Office of Nuclear Physics under Grant No. DE-FG02-94ER40818.
[1] Z. Hadzibabic et al., Nature (London) 441, 1118 (2006).

[2] V. Schweikhard, S. Tung, and E. A. Cornell, Phys. Rev. Lett. 99, 030401 (2007).

[3] P. Krüger, Z. Hadzibabic, and J. Dalibard, Phys. Rev. Lett. 99, 040402 (2007)

[4] Yu. E. Lozovik and V. I. Yudson, JETP Lett. 22, 274 (1975); Solid State Commun. 19, 391 (1976); Sov. Phys. JETP 44, 389 (1976).

[5] S. I. Shevchenko, Sov. J. Low Temp. Phys. 2, 251 (1976).

[6] See, e.g., J. P. Eisenstein and A. H. MacDonald, Nature (London) 432, 691 (2004), and references therein.

[7] See, e.g., M. Y. Kharitonov and K. B. Efetov, e-print arXiv:0903.4445, and references therein.

[8] D. G. Ravenhall, C. J. Pethick, and J. R. Wilson, Phys. Rev. Lett. 50, 2066 (1983).

[9] M. Hashimoto, H. Seki, and M. Yamada, Prog. Theor. Phys. 71, 320 (1984).
[10] M. Taglieber, A.-C. Voigt, T. Aoki, T. W. Hänsch, and K. Dieckmann, Phys. Rev. Lett. 100, 010401 (2008).

[11] E. Wille et al., Phys. Rev. Lett. 100, 053201 (2008).

[12] A.-C. Voigt et al., Phys. Rev. Lett. 102, 020405 (2009).

[13] V. Efimov, Phys. Lett. B 33, 563 (1970); Nucl. Phys. A 210, 157 (1973).

[14] Y. Nishida and S. Tan, Phys. Rev. Lett. 101, 170401 (2008).

[15] Y. Nishida, Ann. Phys. 324, 897 (2009).

[16] M. A. Ruderman and C. Kittel, Phys. Rev. 96, 99 (1954).

[17] T. Kasuya, Prog. Theor. Phys. 16, 45 (1956).

[18] K. Yosida, Phys. Rev. 106, 893 (1957).

[19] N. Read and D. Green, Phys. Rev. B 61, 10267 (2000).

[20] S. Tewari, S. Das Sarma, C. Nayak, C. Zhang, and P. Zoller, Phys. Rev. Lett. 98, 010506 (2007).

[21] N. Prokof'ev, O. Ruebenacker, and B. Svistunov, Phys. Rev. Lett. 87, 270402 (2001); N. Prokof'ev and B. Svistunov, Phys. Rev. A 66, 043608 (2002). 